\title{
Internal energy dependence in x-ray-induced molecular fragmentation: An experimental and theoretical study of thiophene
}

\author{
E. Kukk, ${ }^{1, *}$ D. T. Ha, ${ }^{2}$ Y. Wang, ${ }^{2,3}$ D. G. Piekarski, ${ }^{2}$ S. Diaz-Tendero, ${ }^{2}$ K. Kooser, ${ }^{1}$ E. Itälä, ${ }^{1}$ H. Levola, ${ }^{1}$ \\ M. Alcamí, ${ }^{2}$ E. Rachlew, ${ }^{4}$ and F. Martín ${ }^{2,3,5}$ \\ ${ }^{1}$ Department of Physics and Astronomy, University of Turku, FIN-20014 Turku, Finland \\ ${ }^{2}$ Departamento de Química, Módulo 13, Universidad Autónoma de Madrid, Cantoblanco 28049 Madrid, Spain \\ ${ }^{3}$ Instituto Madrileño de Estudios Avanzados en Nanociencia, Cantoblanco, 28049 Madrid, Spain \\ ${ }^{4}$ Atomic and Molecular Physics, Royal Institute of Technology KTH, Stockholm, Sweden \\ ${ }^{5}$ Condensed Matter Physics Center (IFIMAC), Universidad Autónoma de Madrid, 28049 Madrid, Spain
}

(Received 10 February 2015; published 24 April 2015)

\begin{abstract}
A detailed experimental and theoretical investigation of the dynamics leading to fragmentation of doubly ionized molecular thiophene is presented. Dissociation of double-ionized molecules was induced by $\mathrm{S} 2 p$ core photoionization and the ionic fragments were detected in coincidence with Auger electrons from the core-hole decay. Rich molecular dynamics was observed in electron-ion-ion coincidence maps exhibiting ring breaks accompanied by hydrogen losses and/or migration. The probabilities of various dissociation channels were seen to be very sensitive to the internal energy of the molecule. Theoretical simulations were performed by using the semiempirical self-consistent charge-density-functional tight-binding method. By running thousands of these simulations, the initial conditions encountered in the experiment were properly taken into account, including the systematic dependencies on the internal (thermal) energy. This systematic approach, not affordable with first-principle methods, provides a good overall description of the complex molecular dynamics observed in the experiment and shows good promise for applicability to larger molecules or clusters, thus opening the door to systematic investigations of complex dynamical processes occurring in radiation damage.
\end{abstract}

DOI: 10.1103/PhysRevA.91.043417

PACS number(s): $33.80 . \mathrm{j}, 34.10 .+\mathrm{x}$

\section{INTRODUCTION}

Following the dynamics of molecular reactions as they proceed has been a topic of keen interest for chemists as well as physicists, from both the fundamental and applied points of view [1-4]. Unimolecular reactions that follow the deposition of energy into the system by photoabsorption represent a particular category that has recently spurred significant theoretical and experimental efforts [4-10]. Such radiationinduced processes are omnipresent: they are encountered in the atmosphere, interstellar matter, living tissues, and materials exposed to $\mathrm{x}$ rays or UV radiation. The reason for the increased interest is twofold - on one hand the development of instrumentation and the appearance of new experimental tools such as free-electron lasers provide comprehensive high-quality data; on the other hand the latest theoretical methods coupled with the ever-increasing computer capacity have become capable of modeling molecular dynamics (MD) at a level that can potentially reproduce the experimental observables in close detail [11-13].

Despite active studies, our understanding of MD in radiation-induced reactions is still very limited, particularly when it comes to medium- and large-sized systems (typically with more than ten atoms). For these systems, thermodynamic and statistical methods, in which dynamics is ignored, are common approaches. In contrast, for small molecules, accurate tracking of the combined classical motion of the nuclei

\footnotetext{
*edwin.kukk@utu.fi; Turku University Centre for Materials and Surfaces (MATSURF).
}

and the quantum motion of electrons is possible and has been successfully accomplished $[13,14]$, e.g., by using firstprinciples time-dependent density-functional theory (DFT) and/or Born-Oppenheimer (BO) MD methods for dynamics initiated from, respectively, the excited and ground states of the molecule. Ideally, similar first-principles methods should be used for medium- and large-sized systems; however, due to their high computational cost, they are unpractical for reproducing the MD resulting from a statistically meaningful sampling of initial conditions such as those required to interpret experiments. Thus, simpler MD methods are mandatory.

In the present study, we investigate a representative medium-sized molecule, thiophene $\left(\mathrm{C}_{4} \mathrm{H}_{4} \mathrm{~S}\right)$, applying both experimental and computational methods. Thiophene is a very suitable target due to its rigid five-member aromatic ring and the lack of side chains and of room-temperature isomers. The experimental results show that, following x-ray ionization, thiophene undergoes a very rich MD, which is fully characterized by using high-resolution multicoincidence detection. The observed MD involves numerous ring ruptures accompanied by hydrogen loss and/or migration. Furthermore, with the ability of the experiment to accurately choose the internal energy and the particular electronic state of the parent dication, we demonstrate the high sensitivity of the dissociation patterns to these conditions. In combination with extensive MD simulations, which account for a sampling of initial conditions, we determine why the observed dynamics is sensitive to the different experimental parameters and what is the underlying physical quantity or condition that is the important parameter. For example, is it sufficient to know 
just the internal energy of the molecule or is it important to accurately account for the electron motion in various electronic states? The present study offers an answer to these questions.

\section{EXPERIMENT}

In our experiment, we make use of coincident detection, in which several particles from a sequence of quantum events are observed and the interdependencies of their properties can thus be determined. Here, we employ electron-energyresolved (photo)electron-(photo)ion-(photo)ion coincidence (often referred to as PEPIPICO) spectroscopy [3,5,15-18]. In this PEPIPICO experiment, we used a custom-made Wiley-McLaren-type ion time-of-flight (TOF) spectrometer and a hemispherical electron energy analyzer (Omicron EA125) with $125 \mathrm{~mm}$ nominal radius. The electron analyzer was reequipped with a fast position-sensitive resistive anode readout based on a 40-mm-active-diameter microchannel plate detector (Quantar Inc.). Also new electronics and software for recording the electron detector signal and controlling electron lens and hemisphere electrodes was developed, resulting in enhanced transmission of the analyzer and better optimization options. More details on the coincidence setup can be found in Ref. [19]. The experiment was performed at the soft X-ray beamline I411 at the MAX-II synchrotron [20], using monochromatized radiation of $191 \mathrm{eV}$ photon energy. The electron analyzer was operated at $200 \mathrm{eV}$ pass energy using $1 \mathrm{~mm}$ entrance slit, which provided instrumental resolution of about $0.77 \mathrm{eV}$ (FWHM). During the coincidence measurement with the $\mathrm{S} L V V$ Auger electrons, the analyzer was tuned to capture the kinetic energy window from 120 to $150 \mathrm{eV}$. The kinetic energy scale of the analyzer was calibrated using the known line energies in the Ar LMM Auger spectrum.

The ion TOF spectrometer was operated under WileyMcLaren conditions, applying $-450 \mathrm{~V}$ potential to the drift tube and $\pm 75 \mathrm{~V}$ to the ion extraction grids. The start triggers for the ion TOF measurement in pulsed extraction field mode were provided by the electron detector. In order to reduce the contribution from so-called "false" coincidences [21], low ionization rates were used with about 20 electrons/s counted. In addition, artificial triggers were generated to record PIPICO events that contain only the false coincidences; these were then used for subtracting false coincidence background from the PEPIPICO maps.

The sequence of events that we concentrated on was initiated by the photoemission of the sulfur $2 p$ electrons by $191 \mathrm{eV}$ photons. Within a few femtoseconds, the $2 p$ vacancy was filled by another electron from any of the molecular orbitals and a second electron was ejected from another or the same orbital. This relaxation process is known as the Auger decay (referred to hereafter as the $L V V$ Auger decay) and the ejected electron as the Auger electron. The doubly charged molecule created is predominantly unstable and dissociates: this is the unimolecular MD studied here. In the experiment, the two resultant charged fragments are detected in coincidence. In addition to their masses and charges, also certain information on the various stages of the MD is conveyed to the observer by the ions' momenta and their correlations $[18,22]$.

\section{THEORY}

Extensive MD simulations have been carried out by using the semiempirical self-consistent charge-density-functional tight-binding (SCC-DFTB) method [23] as implemented in the DFTB + code [24]. In the SCC-DFTB approach used here, the Kohn-Sham energy is expanded to the second order with respect to charge-density fluctuations [23]. The Slater-Koster parameters from the mio-1-1 parameter set $[23,25]$ have been chosen to calculate the electronic and repulsive parts of the DFTB energy. At each step of these simulations, the potential energy is calculated by minimizing the electronic energy self-consistently. The electronic entropy contribution due to the electronic excitation effect is treated using the Fermi-Dirac smearing at the electronic temperature of $1500 \mathrm{~K}$.

In the theoretical approach applied here, the molecule is assumed to be in the electronic ground state and the nuclei move classically in the corresponding ground-state potential energy surface (PES). The main difference from first-principles BO MD methods is the description of the electronic structure, which is performed by using a simple tight-binding approach instead of by solving the exact Kohn-Sham DFT equations. Similarly to first-principles methods, at each time step the potential energy experienced by the nuclei is calculated by selfconsistently minimizing the electronic energy of the system. Altogether over 11000 trajectories were computed, as specified below. Finally, for a better understanding of the dynamics, we have computed the minima and transition states of the PES associated with the relevant fragmentation pathways and exit channels. This has been done at the DFT-B3LYP (Becke, threeparameter, Lee-Yang-Parr) $/ 6-311++\mathrm{G}(d, p)$ level by using GAUSSIAN09 [26].

All MD simulations are carried out in the microcanonical $(N V E)$ ensemble. The Verlet algorithm is utilized for time integration. We have chosen the time step of $0.1 \mathrm{fs}$, which has been tested to give reasonable conservation of the total energy. Various internal energies $(n \times 1000 \mathrm{~K}$, where $n=$ $0,1,2, \ldots, 26)$ were deposited into the parent molecule. At the beginning of the simulation, the internal energy is randomly distributed to all the atoms according to the Boltzmann distribution and can thus be represented as temperature. However, a restriction is added that the total momentum of all atoms is zero and therefore the three translational degrees of freedom do not receive energy (as this would not be available for MD). At low internal energies $(T \leqslant 5000 \mathrm{~K})$ the molecular dynamics is very slow and the trajectories were run until 100 ps, 100 for each temperature. At energies corresponding to $T>5000 \mathrm{~K}, 500$ trajectories were run per temperature point, but they were terminated at less than $40 \mathrm{ps}$, since the MD became much faster owing to the higher energy.

To check the accuracy of the results obtained with the SCC-DFTB method, we have performed a few first-principles MD calculations in a more limited range of internal energies. In particular, we have used the atom-centered density matrix propagation method (ADMP) [27-29] with a B3LYP/6$31 \mathrm{G}(d, p)$ basis set and a fictitious electron mass of $0.22 \mathrm{amu}$. The resulting fragmentation yields are compatible with those from the SCC-DFTB calculations. 


\section{EXPERIMENTAL RESULTS}

In the PEPIPICO measurement, one can detect either the photoelectron or the Auger electron, both providing different type of information. The kinetic energy $E_{\text {kin }}$ of the Auger electron is directly linked to the internal energy of the final dicationic state of the molecule after the Auger process. It is convenient to measure the internal energy relative to the lowest-energy dicationic state:

$$
E_{\text {int }}\left(M^{++}\right)=E_{b}\left(M^{*+}\right)-E_{\text {kin }}-V_{\text {dip }} .
$$

Here, $E_{b}\left(M^{*+}\right)$ denotes the binding energy of the $S 2 p^{-1}$ core hole state and $V_{\text {dip }}$ is the double ionization potential of the molecule. $E_{\text {int }}$ can be contained in both the electronic and the nuclear subsystem. By measuring the Auger electron energy, one is thus able to select molecules with particular internal energy. But the experiment does not tell of the fate of this energy, whether, when, or how it is transferred between the nuclear and electronic systems, and what are the mechanisms through which it acts on the MD.

The results of the PEPIPICO measurements following the $\mathrm{S}$ $2 p$ ionization and Auger decay are summarized in the ion-ion coincidence map in Fig. 1, which is a $2 \mathrm{D}$ histogram constructed from about $10^{6}$ individual quantum events. The details of creating and interpreting such maps can be found, e.g., in Ref. [30]. Briefly, the flight times (TOFs) of the two ions arriving as a pair are plotted versus each other, the faster ion's TOF as the $X$ and the slower ion's as the $Y$ coordinate. The color coding of the intensity scale was chosen such as to emphasize weak patterns.

The TOFs identify the masses of the two fragments, since the time of flight $\sim \sqrt{(M / Q)}$. In reality, the TOF pairs are spread along narrow tilted patterns due to the anticorrelation of the ion momenta [18,22]. Each PEPIPICO pattern represents a particular dissociation channel; in Fig. 1 they are grouped together according to the aromatic-ring-breaking pattern that

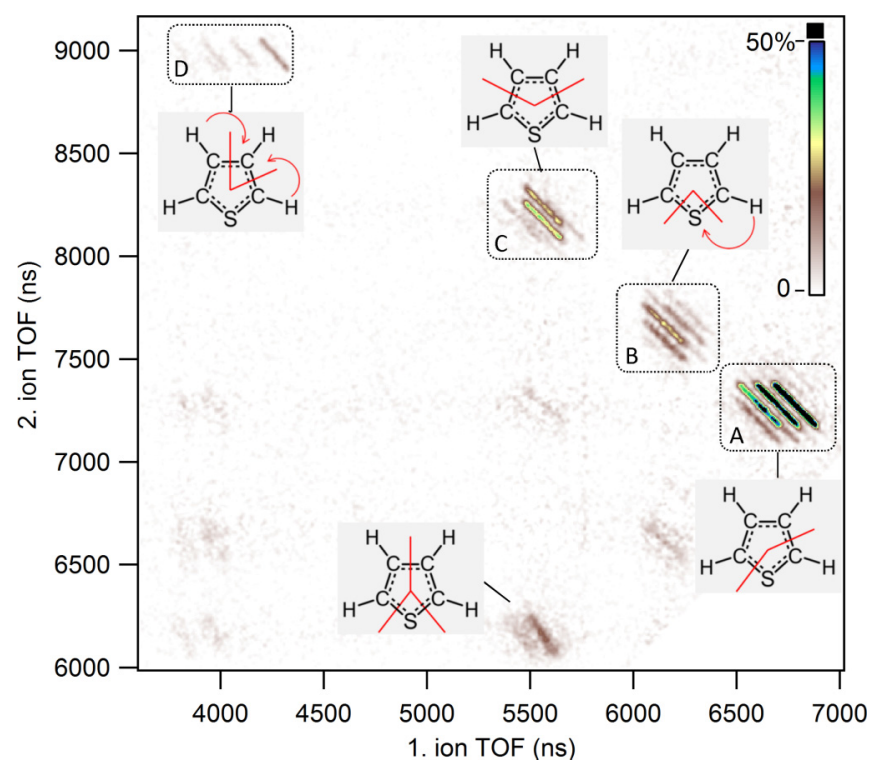

FIG. 1. (Color online) PEPIPICO map of fragment ion pairs measured in coincidence with sulfur LVV Auger electrons of thiophene within the kinetic energy range from 120 to $150 \mathrm{eV}$. they represent. Within the groups A-D, the individual patterns are distinguished by the possible loss of hydrogens from one or both fragments. The overall MD in this experiment is dominated by essentially two-body ring breakups with associated hydrogen dynamics. The strongest group $\mathrm{A}$, for example, represents the process $\mathrm{C}_{4} \mathrm{H}_{4}{ }^{+} \mathrm{S} \rightarrow \mathrm{C}_{3} \mathrm{H}_{n}{ }^{+}+$ $\mathrm{CSH}_{m}{ }^{+}, n=1-3, m=0,1$. (Note that $\mathrm{CS}^{+}$is not observed together with $\mathrm{C}_{3} \mathrm{H}_{3}{ }^{+}$.) The only pattern with multiple ring fragments that has significant intensity in Fig. 1 corresponds to the ions $\mathrm{C}_{2} \mathrm{H}_{2}{ }^{+}$and $\mathrm{S}^{+}$. There are also possible channels involving the $\mathrm{H}^{+}$fragment, which were outside the recorded TOF window. However, the hydrogen ions are not expected to be abundant, as they would also distort the momentum distributions of the observed heavier ions and this is not seen. Figure 1 is a summary of all observed events and does not differentiate according to the key property: the electron energy. The PEPIPICO events can be sorted according to the coincident Auger electron's energy and the number of events in the PEPIPICO patterns counted for every energy bin. We refer to the resulting curves as the (photo)ion-(photo)ion yields (PIPIYs) and they show how the branching ratios among
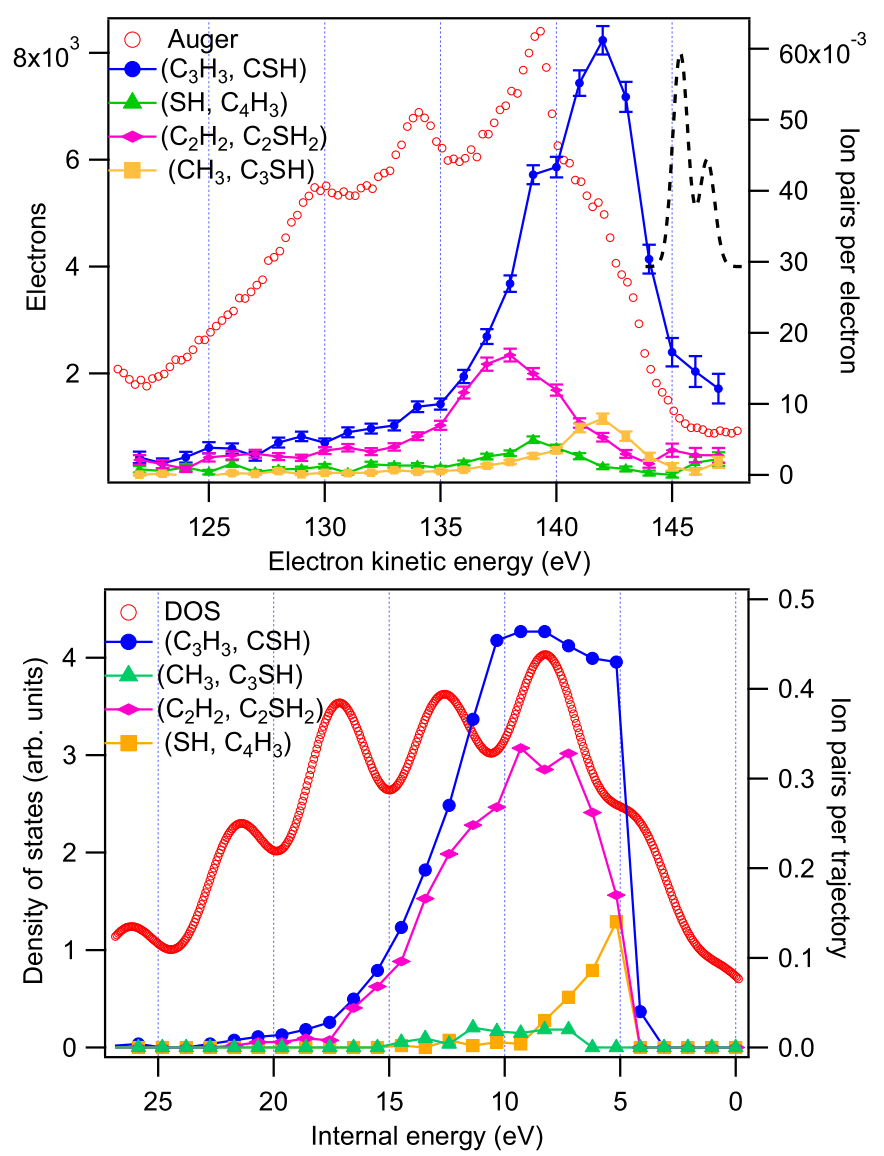

FIG. 2. (Color online) Top: Sulfur $L V V$ Auger electron spectrum and the PIPIY curves of observed two-body dissociation channels in groups A-D. The dotted line shows the S $2 p$ spin-orbit-split levels convoluted by the lifetime width and the instrument function. Bottom: The density of states (DOS) for the Auger transitions and the calculated PIPIY curves. The PIPIY pair counts are divided by the number of detected coincident electrons (experiment) and by the number of calculated trajectories (theory) at each energy point. 
the various channels change as a function of the electron energy.

The rich MD of Fig. 1 thus results from an interplay between ring breaks and hydrogen dynamics. We will first address the ring-breaking patterns. The top panel of Fig. 2 shows the PIPIY curves for four observed strictly two-body channels corresponding to various ring ruptures defining the groups A-D. The breakups that appear at the lowest internal energy (highest kinetic energy) are $\mathrm{C}_{4} \mathrm{H}_{4} \mathrm{~S}^{2+} \rightarrow \mathrm{C}_{3} \mathrm{H}_{3}{ }^{+}+\mathrm{CSH}^{+}$(in group A) and $\mathrm{C}_{4} \mathrm{H}_{4} \mathrm{~S}^{2+} \rightarrow \mathrm{CH}_{3}{ }^{+}+\mathrm{C}_{3} \mathrm{SH}^{+}$(in group D). Remarkably, however, the latter channel is much weaker. At higher internal (lower kinetic) energies, two other twobody channels open up. At even higher energies, all twobody channels are quenched by the increasing probability of hydrogen losses. A feature not visible in PIPIYs or in PEPIPICO maps is the production of the doubly ionized parent ion $\mathrm{C}_{4} \mathrm{SH}_{4}{ }^{2+}$ and that accompanied by neutral hydrogen loss(es). The experiment shows, however, sharp single-ion yield (PIY) for $\mathrm{C}_{4} \mathrm{SH}_{4}{ }^{2+}$ in the range $E_{\text {kin }}=142.5-146 \mathrm{eV}$, confirming that molecular dications with $E_{\text {int }}$ less than about 5 $\mathrm{eV}$ are stable. There are also weak ion yields of $\mathrm{C}_{4} \mathrm{SH}_{2}{ }^{2+}$ and $\mathrm{C}_{4} \mathrm{SH}^{2+}$ below $E_{\text {kin }}=145 \mathrm{eV}$ over a broad $E_{\text {kin }}$ range.

\section{THEORETICAL SIMULATIONS AND DISCUSSION}

\section{A. Ring breakup patterns and hydrogen dynamics}

The results of the MD simulations are shown in the lower panel of Fig. 2. First, it shows the calculated density of two-hole states (DOS) that results from filling the $\mathrm{S} 2 p$ orbital by an electron and ejection of another electron from the singly charged molecule with energy $E_{\text {kin }}$. The DOS curve is given in order to illustrate the contributions of various internal energies $\left(E_{\text {int }}\right)$ to the total fragmentation pattern of the molecule. Note that the scales of both panels are directly comparable and are linked by Eq. (1). Final two-hole $(i, j)$ state energies for the DOS curve were obtained simply as sums of the Kohn-Sham orbital energies $E_{i}$ and $E_{j}$ of the neutral molecule. In matching the simulation's energy scale with the experiment, $E_{\text {kin }}=-E_{2 p}+E_{i}+E_{j}$, where $E_{2 p}=-161.25$ $\mathrm{eV}$ is the orbital energy of S $2 p$. The calculated DOS captures the main structure of the measured Auger spectrum quite well. The $E_{\text {int }}=0$ position as obtained from experimental $V_{\text {dip }}$ and $E_{b}\left(M^{*+}\right)$ values is indicated by the dotted curve in the top panel [31]. Figure 2 also shows that the calculated PIPIYs catch the essential features observed in the experiment: the dominance of the two-body channels in groups A and C (Fig. 1) over the two-body channels in B and D. Notably, A and C do not involve any hydrogen migration, while $\mathrm{B}$ requires one and $\mathrm{D}$ two hydrogen migrations. This combined dynamics is also correctly captured by the simulations, illustrated also by the two animations given as Supplemental Material [32]. Hydrogen migration in similar molecules has also been observed in other contexts $[13,33]$.

\section{B. Potential energy surface}

For a better understanding of the relative weights of the observed ring breakup patterns, we have computed the minima and transition states of the potential energy surface of doubly ionized thiophene by using density functional theory with an extended B3LYP/6-311++g $(d, p)$ basis set. The relevant critical points are shown in Fig. 3. In the left panel, minima corresponding to the different two-body fragmentation channels and the highest transition states connecting those minima by lowest-energy pathways are shown. In the left panel,
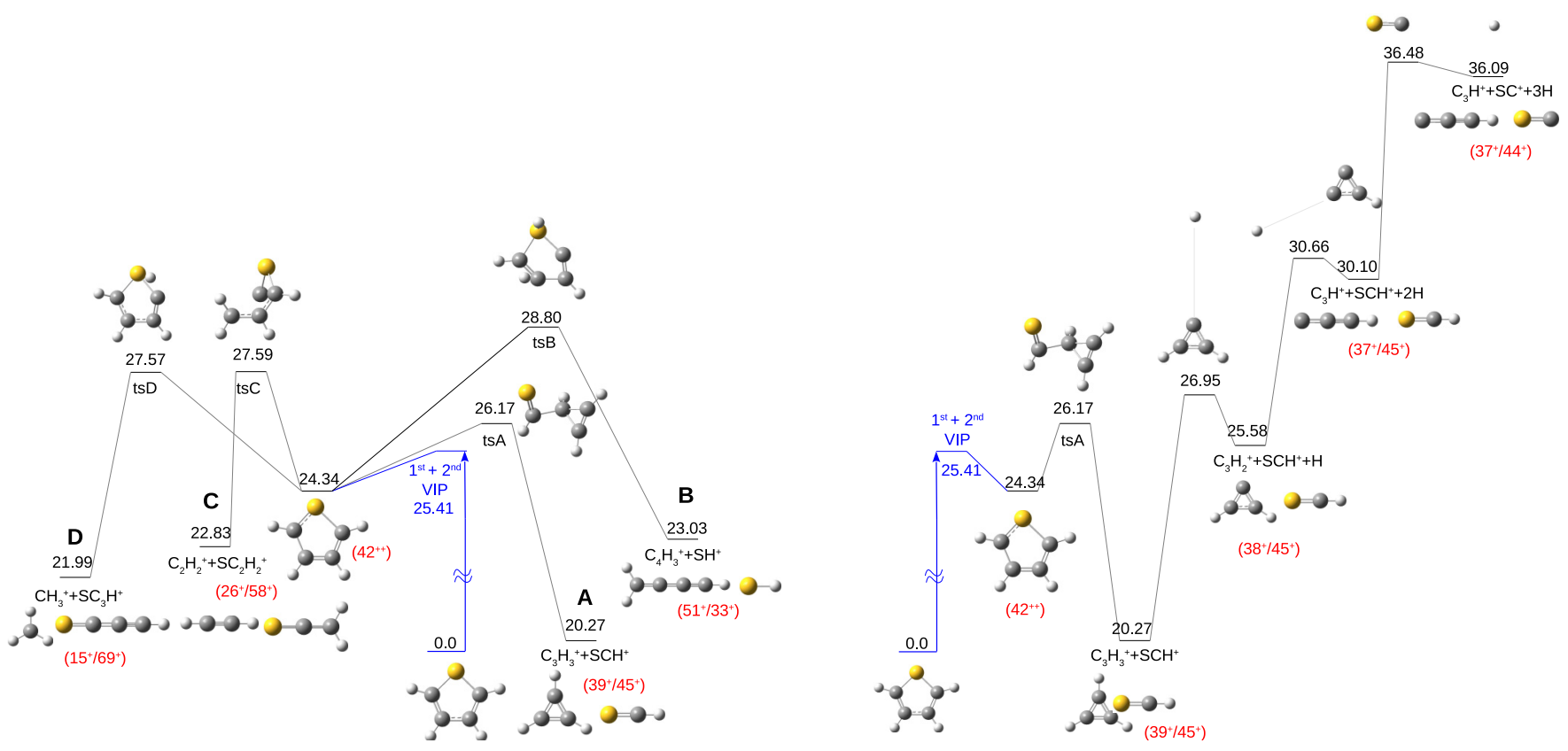

FIG. 3. (Color online) Left panel: PES critical points connecting the two-body fragmentation channels of doubly ionized thiophene; the letters A, B, C, and D refer to the groups defined in Fig. 1. Right panel: PES critical points connecting hydrogen emission steps in group A. Relative energy (in eV) for the minima and transition states is given with respect to the neutral molecule in its groud state. "1st + 2nd VIP" is the double vertical ionization potential (i.e., in a Frank-Condon transition). 
the minima corresponding to the products in the different two-body fragmentation channels are shown. The pathways leading to these fragments are quite complex with several minima and transition states. In the simplified presentation, we show only the transition states in each path that appear at the highest energy (bottleneck in the PES). In the right panel, minima and transition states for the different hydrogen emissions in group A are shown.

The dominance of the two-body channel in group A for all internal energies (Fig. 2) can be easily understood in terms of thermodynamical and statistical arguments. Indeed this channel leads to the most stable fragmentation products $\left(\mathrm{C}_{3} \mathrm{H}_{3}{ }^{+}+\mathrm{CSH}^{+}\right)$and proceeds through the lowest energy barrier. Similar arguments suggest that the two-body channel in $\mathrm{D}$ should be next in importance because the corresponding products follow in stability $\left(\mathrm{CH}_{3}{ }^{+}+\mathrm{C}_{3} \mathrm{SH}^{+}\right)$and the fragmentation dynamics goes through the second-lowest energy barrier. However, two-body fragmentation in D is much less important than in $\mathrm{C}$ and as important as in $\mathrm{B}$, which respectively involve products $\simeq 0.8$ and $1.0 \mathrm{eV}$ less stable than in $\mathrm{D}$ and barriers 0.02 and $1.3 \mathrm{eV}$ higher (see Fig. 3). Consequently, in this case, the observed patterns can only be explained in terms of dynamical effects that reflect the complexity of the PES landscape.

\section{Fragmentation time scales and sequences}

A statistical analysis of over 1100 trajectories shows that fragmentation time scales are heavily influenced by the amount of internal energy, even within a particular fragmentation channel. Figure 4 shows average times of three different kinds of fragmentation events: first hydrogen(s) ejection, ring opening, and ring fragmentation of groups $\mathrm{A}, \mathrm{B}, \mathrm{C}$, and $\mathrm{D}$ as a function of internal energy. The average times of first hydrogen(s) ejection correspond to ejection of the first hydrogen, either in the form of atomic $\mathrm{H}$ or in the form of an $\mathrm{H}_{2}$ molecule. Ring opening times indicate the instant at which the first ring bond(s) (either C-C or C-S) breaks. Ring fragmentation times indicate when the molecular ring dissociates into at least two fragments. The criterion used to decide that a bond is broken is that the distance between the two atoms forming that bond is larger than $2 \AA$. Error bars represent the standard deviation with respect to the mean values.

The two animations provided as Supplemental Material [32] show fragmentation processes of channels resulting in cation pairs $\left(\mathrm{CH}_{3}{ }^{+}, \mathrm{C}_{3} \mathrm{SH}^{+}\right)$and $\left(\mathrm{C}_{3} \mathrm{H}^{+}, \mathrm{CSH}^{+}\right)$. Details of the animation layout are given in [32]. They show how, at the higher internal energies, the ring opens in 10-20 fs and separates into two fragments further few tens of femtoseconds later (except for group D, where ring opening and fragmentation occur almost simultaneously). At the lower internal energies, both processes are substantially delayed: ring opening occurs after a few picoseconds and fragmentation a few picoseconds later.

\section{Hydrogen loss dynamics}

Let us now focus on the hydrogen loss dynamics within a group. Figure 5 depicts the PIPIY curves obtained from experiment (top) and theory (bottom) for the patterns within group A-the $\mathrm{C}_{3} \mathrm{H}_{n}{ }^{+}+\mathrm{CSH}_{m}{ }^{+}$fragmentation channels. As
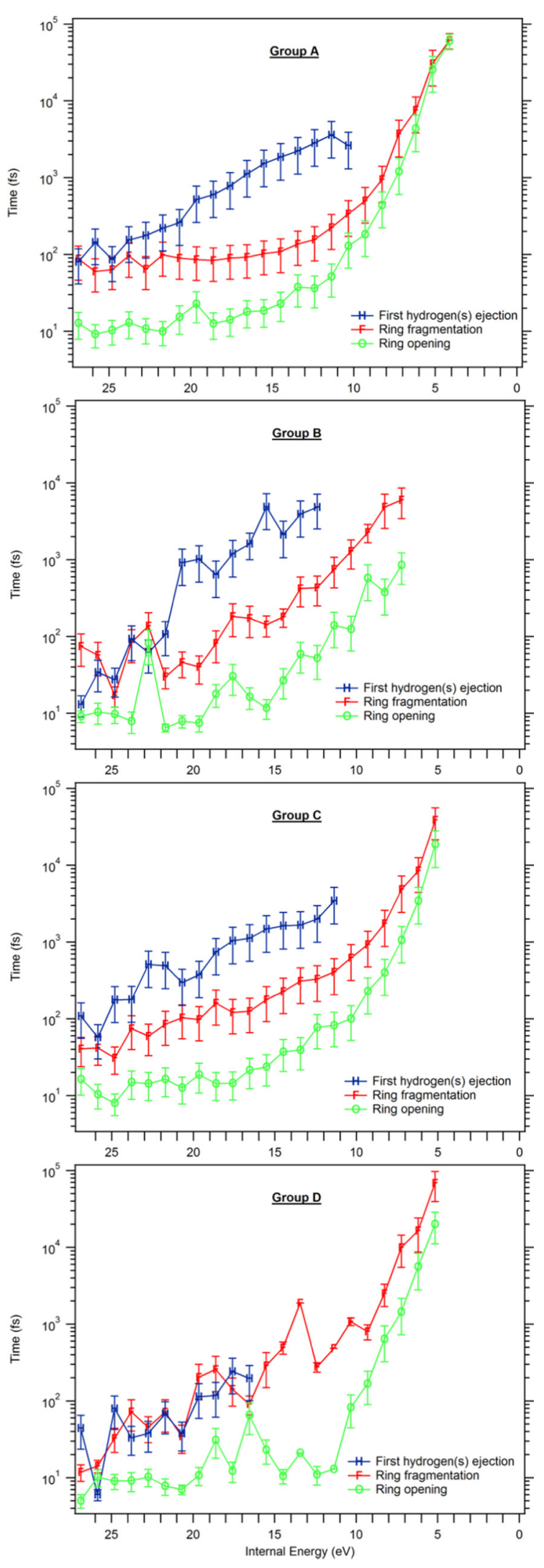

FIG. 4. (Color online) Average times of various fragmentation events in the dissociation processes of groups A, B, C, and D as a function of internal energy. Refer to the text for description of the fragmentation events. The error bars correspond to standard deviations. 

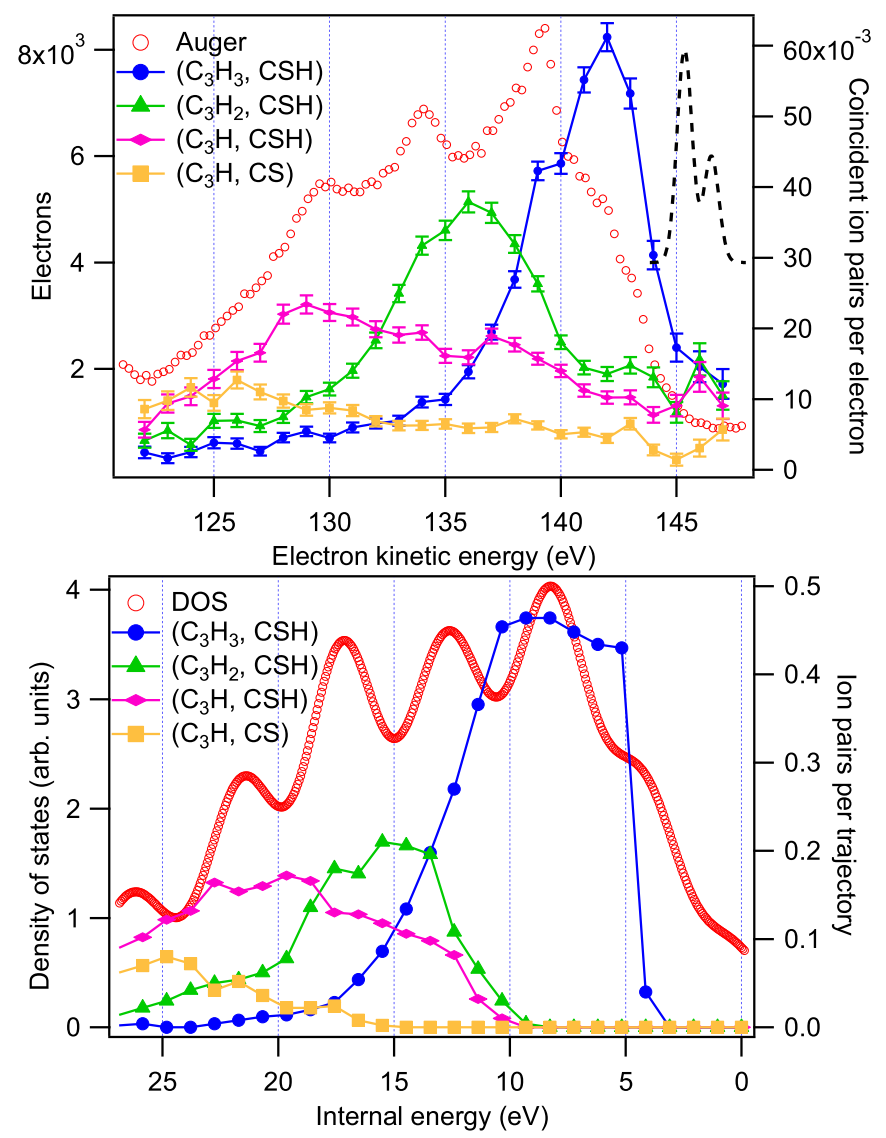

FIG. 5. (Color online) Sulfur $L V V$ Auger electron spectrum and the PIPIY curves (normalized by the Auger electron counts) of the dissociation channel $\left(\mathrm{CSH}^{+}, \mathrm{C}_{3} \mathrm{H}_{3}{ }^{+}\right)$and its accompanying hydrogen loss channels.

can be seen, hydrogen loss is a process that strongly depends on energy. Both experiment and theory demonstrate that, as internal energy increases, the number of hydrogen atoms that are lost from the $\mathrm{C}_{3} \mathrm{H}_{3}{ }^{+}$fraction increases, so that $\mathrm{C}_{3} \mathrm{H}_{2}{ }^{+}$ and then $\mathrm{C}_{3} \mathrm{H}^{+}$fragments become subsequently dominant. The loss of the third hydrogen occurs from the $\mathrm{CSH}^{+}$not the $\mathrm{C}_{3} \mathrm{H}^{+}$fraction of the ring. The first hydrogen loss requires approximately $6 \mathrm{eV}$ extra internal energy (Fig. 5, top). This value is overestimated in the MD calculations (bottom), but it is very similar to the energy required to go from the $\mathrm{C}_{3} \mathrm{H}_{n}{ }^{+}$ minimum to $\mathrm{C}_{3} \mathrm{H}_{n-1}+$ in the PES. The second $\mathrm{H}$ loss starts at about the same energies, but has a slow buildup too, at around $E_{\text {kin }} \approx 130 \mathrm{eV}$, becoming the dominant channel in group $\mathrm{A}$. The third $\mathrm{H}$ ejection requires again considerable extra energy. Overall, hydrogen loss from the $\mathrm{CSH}^{+}$fraction is rather unlikely in the whole range of internal energies. All these features are in excellent agreement with theory, the only discrepancy being the above-mentioned overestimation of the first $\mathrm{H}$ loss. A similar behavior is observed for the individual patterns contained in groups $\mathrm{B}, \mathrm{C}$, and $\mathrm{D}$ of Fig. 1.

According to our simulations, the first hydrogen ejection (either atomic $\mathrm{H}$ or molecular $\mathrm{H}_{2}$ ) occurs after the two-body fragmentation of the ring, either immediately after or hundreds of femtoseconds later. There does not seem to be a preference for either a sequential or a concerted process. If a neutral fragment (such as $\mathrm{H}$ or $\mathrm{H}_{2}$ ) were to be ejected from a charged one in a secondary, time-delayed process, it would cause a slight but observable change to the $-45^{\circ}$ tilt angle of the patterns observed in the PEPIPICO map. However, all patterns in groups $\mathrm{A}-\mathrm{D}$ exhibit a $-45^{\circ}$ angle within error limits, which is consistent with the theoretical findings-randomly timed hydrogen loss would mostly cause a slight broadening to the $-45^{\circ}$ patterns.

\section{CONCLUSIONS}

In conclusion, we have shown how electron-energyresolved multiparticle coincidence experiments provide very detailed and accurate information on the rich molecular dynamics observed in the photodissociation of medium-sized molecules such as doubly ionized thiophene. The branching ratios of the numerous dissociation channels are highly sensitive to the molecular internal energy across the whole energy range of molecules prepared by the Auger decay of a core vacancy. Theoretical SCC-DFTB simulations, based on a meaningful statistical sample of trajectories, reproduce all the essential features of this dynamics - the ring-breaking patterns, their energy dependence and relative weights, the hydrogen migration and ejection, their probabilities, and also, qualitatively, energy dependence. Notably, the theoretical modeling did not need to take into account at all the specifics of the electron distribution in the Auger final states at the start of dissociation, nor the localization of the initial core hole. Quite good agreement with experiment was achieved with only the internal energy (temperature) as a parameter. This suggests (i) a very rapid (of the order of a few femtoseconds) redistribution of the excitation energy among all molecular sites in agreement with recent experimental and theoretical work [34-36], i.e., no selectivity in the particular holes that are created, (ii) a similarly fast Auger decay, and (iii) a classical behavior of nuclei, except maybe protons.

Having demonstrated its usability for medium-sized molecules, the present theoretical approach looks very promising for studying larger systems, e.g., small nucleoside-water clusters that are closer to the cell environment than individual molecules, but for which unequivocal interpretation of experimental results without theoretical input would be impossible.

\section{ACKNOWLEDGMENTS}

This work was supported by a grant of the Academy of Finland, Finnish Cultural Foundation, MICINN Project No. FIS2010-15127 and ERA-Chemistry Project No. PIM2010EEC-00751, the MINECO Projects No. FIS201342002-R and No. CTQ2013-43698-P, the European COST Action No. CM1204 XLIC, an Advanced Grant of the European Research Council XCHEM No. 290853, the CAM Project NANOFRONTMAG, and the European Grant MCITN CORINF. We acknowledge allocation of computer time at the Centro de Computación Científica of the Universidad Autónoma de Madrid (CCC-UAM), the Barcelona Supercomputer Center, and by the FGI project (Finland). S.D.-T. acknowledges the "Ramón y Cajal" Program. 
[1] L. R. Khundkar and A. H. Zewail, Annu. Rev. Phys. Chem. 41, 15 (1990).

[2] P. Casavecchia, Rep. Prog. Phys. 63, 355 (2000).

[3] Frontiers of Coincidence Experiments, edited by K. Ueda (Elsevier, Amsterdam, 2004).

[4] J. Ullrich, A. Rudenko, and R. Moshammer, Annu. Rev. Phys. Chem. 63, 635 (2012).

[5] N. Saito, X. J. Liu, Y. Morishita, I. H. Suzuki, and K. Ueda, J. Electron Spectrosc. Relat. Phenom. 156-158, 68 (2007).

[6] G. Prümper, X. Liu, H. Fukuzawa, K. Ueda, V. Carravetta, J. Harries, Y. Tamenori, and S. Nagaoka, J. Phys.: Conf. Series 88, 012008 (2007).

[7] O. Plekan, V. Feyer, R. Richter, M. Coreno, M. De Simone, and K. C. Prince, Chem. Phys. 334, 53 (2007).

[8] M. Casida and M. Huix-Rotllant, Annu. Rev. Phys. Chem. 63, 287 (2012).

[9] D. T. Ha, Y. Wang, M. Alcami, E. Itälä, K. Kooser, S. Urpelainen, M. A. Huels, E. Kukk, and F. Martin, J. Phys. Chem. A 118, 1374 (2014).

[10] B. Erk, R. Boll, S. Trippel, D. Anielski, L. Foucar, B. Rudek, S. W. Epp, R. Coffee, S. Carron, S. Schorb et al., Science 345, 288 (2014).

[11] P. López-Tarifa, M.-A. H. du Penhoat, R. Vuilleumier, M.-P. Gaigeot, I. Tavernelli, A. Le Padellec, J.-P. Champeaux, M. Alcamí, P. Moretto-Capelle, F. Martín et al., Phys. Rev. Lett. 107, 023202 (2011).

[12] M. E.-A. Madjet, O. Vendrell, and R. Santra, Phys. Rev. Lett. 107, 263002 (2011).

[13] S. Maclot, D. G. Piekarski, A. Domaracka, A. Mry, V. Vizcaino, L. Adoui, F. Martín, M. Alcamí, B. A. Huber, P. Rousseau et al., J. Phys. Chem. Lett. 4, 3903 (2013).

[14] M. E. Corrales, J. González-Vázquez, G. Balerdi, I. R. Solá, R. de Nalda, and L. Bañares, Nat. Chem. 6, 785 (2014).

[15] C. J. Danby and J.-D. Eland, Int. J. Mass Spectrom. Ion Phys. 8, 153 (1972).

[16] T. A. Field and J. H. Eland, Meas. Sci. Technol. 9, 922 (1998).

[17] R. E. Continetti, Annu. Rev. Phys. Chem. 52, 165 (2001).

[18] A. Hitchcock and J. Neville, in Chemical Applications of Syncrotron Radiation: I, edited by T. Sham (World Scientific, Singapore, 2002).

[19] E. Kukk, R. Sankari, M. Huttula, A. Sankari, H. Aksela, and S. Aksela, J. Electron Spectrosc. Relat. Phenom. 155, 141 (2007).
[20] M. Bässler, A. Ausmees, M. Jurvansuu, R. Feifel, J.-O. Forsell, P. de Tarso Fonseca, A. Kivimäki, S. Sundin, S. Sorensen, R. Nyholm et al., Nucl. Instrum. Methods Phys. Res. Sect. A 469, 382 (2001).

[21] G. Prümper and K. Ueda, Nucl. Instrum. Methods .Phys. Res. Sect. A 574, 350 (2007).

[22] E. Itälä, K. Kooser, E. Rachlew, M. A. Huels, and E. Kukk, J. Chem. Phys. 140, 234305 (2014).

[23] M. Elstner, D. Porezag, G. Jungnickel, J. Elsner, M. Haugk, T. Frauenheim, S. Suhai, and G. Seifert, Phys. Rev. B 58, 7260 (1998).

[24] B. Aradi, B. Hourahine, and T. Frauenheim, J. Phys. Chem. A 111, 5678 (2007).

[25] T. Niehaus, M. Elstner, T. Frauenheim, and S. Suhai, J. Mol. Struct.: THEOCHEM 541, 185 (2001), http://www. sciencedirect.com/science/article/pii/S0166128000007624.

[26] M. J. Frisch, G. W. Trucks, H. B. Schlegel, G. E. Scuseria, M. A. Robb, J. R. Cheeseman, G. Scalmani, V. Barone, B. Mennucci, G. A. Petersson et al., Gaussian 09 Revision B.01 (2010) (Gaussian Inc., Wallingford, CT, 2010).

[27] H. B. Schlegel, J. M. Millam, S. S. Iyengar, G. A. Voth, A. D. Daniels, G. E. Scuseria, and M. J. Frisch, J. Chem. Phys. 114, 9758 (2001).

[28] S. S. Iyengar, H. B. Schlegel, J. M. Millam, G. A. Voth, G. E. Scuseria, and M. J. Frisch, J. Chem. Phys. 115, 10291 (2001).

[29] H. B. Schlegel, S. S. Iyengar, X. Li, J. M. Millam, G. A. Voth, G. E. Scuseria, and M. J. Frisch, J. Chem. Phys. 117, 8694 (2002).

[30] E. Itälä, E. Kukk, D. T. Ha, S. Granroth, A. Calo, L. Partanen, H. Aksela, and S. Aksela, J. Chem. Phys. 131, 114314 (2009).

[31] A few-eV shift in the calculated value in the lower panel of Fig. 2 is quite expected since orbital relaxation is neglected.

[32] See Supplemental Material at http://link.aps.org/supplemental/ 10.1103/PhysRevA.91.043417 for videos of molecular dynamics.

[33] S. De, J. Rajput, A. Roy, P. N. Ghosh, and C. P. Safvan, Phys. Rev. Lett. 97, 213201 (2006).

[34] F. Calegari, D. Ayuso, A. Trabattoni, L. Belshaw, S. De Camillis, S. Anumula, F. Frassetto, L. Poletto, A. Palacios, P. Decleva et al., Science 346, 336 (2014).

[35] F. Remacle and R. D. Levine, Proc. Natl. Acad. Sci. U.S.A. 103, 6793 (2006).

[36] A. I. Kuleff and L. S. Cederbaum, Chem. Phys. 338, 320 (2007). 